\title{
Performance analysis of Adaptive Power Technique for Cooperative Diversity Communications
}

DOI:

10.1109/WCNC.2017.7925946

\section{Document Version}

Accepted author manuscript

Link to publication record in Manchester Research Explorer

\section{Citation for published version (APA):}

Alfitouri, A., \& Hamdi, K. (2017). Performance analysis of Adaptive Power Technique for Cooperative Diversity Communications. In 2017 IEEE Wireless Communications and Networking Conference IEEE.

https://doi.org/10.1109/WCNC.2017.7925946

\section{Published in:}

2017 IEEE Wireless Communications and Networking Conference

\section{Citing this paper}

Please note that where the full-text provided on Manchester Research Explorer is the Author Accepted Manuscript or Proof version this may differ from the final Published version. If citing, it is advised that you check and use the publisher's definitive version.

\section{General rights}

Copyright and moral rights for the publications made accessible in the Research Explorer are retained by the authors and/or other copyright owners and it is a condition of accessing publications that users recognise and abide by the legal requirements associated with these rights.

\section{Takedown policy}

If you believe that this document breaches copyright please refer to the University of Manchester's Takedown Procedures [http://man.ac.uk/04Y6Bo] or contact uml.scholarlycommunications@manchester.ac.uk providing relevant details, so we can investigate your claim.

\section{OPEN ACCESS}




\title{
Performance analysis of Adaptive Power Technique for Cooperative Diversity Communications
}

\author{
Abdurrahman Alfitouri, and Khairi Ashour Hamdi \\ School of Electrical and Electronic Engineering, \\ The University of Manchester, Manchester, M13 9PL, UK \\ Emails: \{Abdurrahman.alfitouri, k.hamdi\}@manchester.ac.uk
}

\begin{abstract}
Cooperative diversity is a promising technique for use in wireless networks because it can provide diversity gain without the need to install multiple antennas at the source or destination. This paper is concerned with the performance analysis of a dual-hop random access network employed adaptive power technique (APT) relay in cooperative diversity networks. The network considered uses amplify-and-forward relaying, and is the only mean to connect source to its destination. This scheme is compared with such popular schemes as the orthogonal scheme, best relay selection, maximal ratio transmission (MRT) and the optimum combining scheme. A new unified mathematical method is developed, which leads to the derivation of new analytical expressions for the overall spectral-efficiency of the dual-hop random access network over Rayleigh fading channels. Numerical results show that the APT can benefit from an increase in the number of relays and reduction of the noise power at the relay and destination. The results also show that the performance of the APT is comparable to the other cooperative schemes, except the MRT. Furthermore, the new mathematical results obtained for APT have been confirmed by Monte Carlo simulation.

Index Terms-Cooperative diversity, adaptive power technique, moment generating function, relay.
\end{abstract}

\section{INTRODUCTION}

Cooperative diversity network technology, see [1]-[3], is known to be an effective means of high data-rate coverage and a way of coping with the fading of wireless channels, both of which are required for future cellular and ad-hoc wireless communication systems. The multiple antennas have been considered as one of cooperative diversity protocols, where it used to increase diversity to combat channel fading [4]. Multiple copies of the data symbols, which lead to improve the system performance, can be obtained at the receiver node, when each antenna at the transmitter sending the same signal information to another antenna at the receiver.

The performance of cooperative diversity using equal gain combinations over Nakagami-m fading channels is proposed in [5]. The single and multiple relay selection schemes and their achievable diversity orders are reported in [6]. The end-to-end performance of a two-hop system with non-regenerative relays over flat Rayleigh-fading channels is proposed in [7]. The average symbol error rate analysis for the distributed spatial diversity wireless system with amplifying relays is presented in [8] for a Rayleigh-fading environment.

Most previous work assumes that the destination has perfect knowledge of the channel for all links. This assumption is not a practical assumption, because the destination cannot obtain the $(S \rightarrow$ Relay) channel information perfectly without noise amplification. Moreover, the complexity overhead for the system will increases with the number of relay nodes [9].

In this paper, we analyse the performance of cooperative diversity networks, where number of relay nodes using to enhance the source signal at the destination. There is one source, one destination, and $\mathrm{M}$ relays can see and communicate to both nodes (source and destination). Each one of these nodes is equipped with single antenna, which can be used for both transmission and reception. There is no direct link between the source and destination nodes. We analyse the performance of adaptive power technique (APT) cooperative diversity networks over Rayleigh fading channels. Each relay needs to know ( $S \rightarrow$ Relays $\rightarrow D$ ) only for its own channels, which makes the complexity of this system less than for the other systems, such as maximal ratio transmission (MRT). The idea is to adaptive the transmitted power for each relay, which leads to optimize the system performance. To the best of the our knowledge, the performance of APT has not previously been addressed in the literature. We derive explicit expressions for the spectral efficiency (SE) of the APT scheme of this model, which can be used to estimate the throughput of the cooperative diversity, and to study the impact of the different system parameters on their efficiency, where the new results expressed in terms of the weights and abscissas of a Laguerre orthogonal polynomial.

The signal-to-noise ratio (SNR) in this model is a ratio of a large number of random variables. However, the direct approach to find the overall SE may require at least $(4 M)$ fold numerical integrations, which is difficult to obtain in general. As result, we investigate the use of a simple and useful Lemma1 to evaluate this averaging, where the result includes a simple mathematical equation that can be easily evaluated. We compare our scheme with some popular schemes, such as the orthogonal scheme (OS), best relay selection (BRS), MRT, and the optimum combining scheme (OCS). Furthermore, simulation results are provided to validate our analysis. We found that the APT can benefit from increase in the number of relays, and the reduction of the noise power at the relay and destination. Also, our results show that the performance of the APT is comparable to the other cooperative schemes performance, except the MRT, whose performance was better.

The remainder of this paper is organized as follows. Section II introduces the system model under investigation for the 


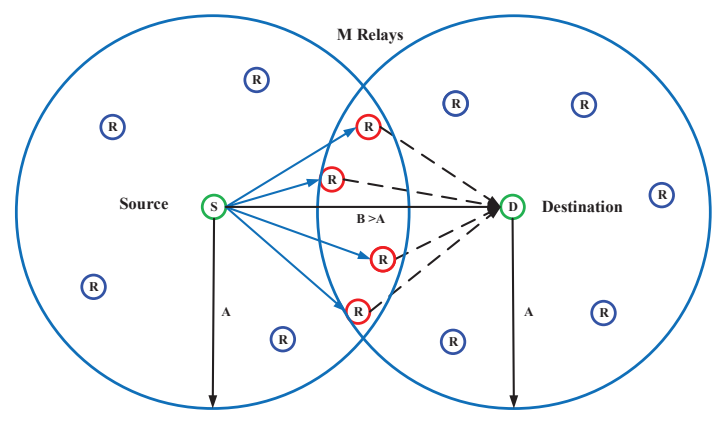

Fig. 1: System Model

EGT, and the other schemes that we are planning to compare with APT. The SE analysis for APT is presented in section III. Results are presented and discussed in Section IV. Finally, concluding remarks are given in Section V.

Notations: $E$ [.] denotes the expectation operator, $\mathrm{B}^{*}$ denotes the conjugates of channel $\mathrm{B},|$.$| denotes the absolute$ value, $\operatorname{Re}(\mathrm{B})$ denotes the real of $\mathrm{B}, \max (\mathrm{B})$ denotes to the maximum of $\mathrm{B}$. Also, we use $\sim C N(0,1)$ to stand for a circularly symmetric complex Gaussian distribution with zero mean and variance 1 .

\section{SySTEM MODEL}

The system model under consideration is shown in Fig. 1. There is one source, one destination, and each one of these nodes is equipped with single antenna, which can be used for both transmission and reception. There are number of relays distributed uniformly in the service area around the source and the destination, and the service area is around each node (source and destination) considered as a circle with radius $A$. The source and destination are hidden from each other, which means no direct link between them. Only M relays in the overlapping area between the source and destination can see and communicate with both the source and destination. The communication between the source and the destination is divided into two phases. During the first phase, which known also as the Broadcast Channel (BC) phase, the source sends its signal to the relays simultaneously. In the second phase, called also as the Multiple Access Channel (MAC) phase, the $\mathrm{M}$ relays forward the amplified signal to the destination. The channel from the source to the $i^{t h}$ relay is denoted as $h_{i}$ and the channel from the $i^{t h}$ relay to the destination as $g_{i}$. All channels are assumed to be subject to independent and identically distributed complex Gaussian fading with zero mean and unit variance, e.g., $h_{i}, g_{i} \backsim C N(0,1)$. All the additive white Gaussian noise (AWGN) terms related to all links $(S \rightarrow$ Relays $\rightarrow D)$, assumed to have zero mean and variance $\left(\sigma_{r}^{2}\right.$, and $\sigma_{d}^{2}$, respectively.). Without loss of generality, In the first phase, the received signal at the $i^{t h}$ relay is given by

$$
y_{i}=h_{i} l_{i}^{-\frac{\alpha}{2}} x+n_{i}
$$

where $x$ is the transmitted symbol with unit power, $h_{i}$ is the complex channel gain between the source and relay $i, l_{i}$ is the distance between the source and relay $i, \alpha$ is the path loss exponent, and $n_{i}$ is AWGN at the $i^{\text {th }}$ relay. During the second phase, each relay amplifies $y_{i}$ by multiplying it with $w_{i}$, which is the weight of relay $i$, and broadcasts it to the destination. The received composite signal at the destination in the $\mathrm{BC}$ phase is given by

$$
y_{D}=\sum_{i=1}^{M} g_{i} w_{i} r_{i}^{-\frac{\alpha}{2}}\left(h_{i} l_{i}^{-\frac{\alpha}{2}} x+n_{i}\right)+n_{d}
$$

where $g_{i}$ is the complex channel gain between the destination and relay $i, r_{i}$ is the distance between the relay $i$ and destination, and $n_{d}$ is AWGN at the destination.

\section{A. Adaptive Power Technique}

In cooperative network, MRT is considered as the optimum technique to combine the multiple relays signal, to achieve maximum data rate. However, the complexity of the MRT is higher than any other technique, where each relay in the network needs to know all the channels between the relays, source, and destination. On the other hand, the BRS is considered as one of cooperation schemes that attracts many interests. It usually achieves full diversity with less synchronization or feedback requirements. However, the achievable rate of BRS will be less than the MRT, where only one relay relaying the data between the source and destination. In this regard, APT is introduced as effective scheme to achieve cooperative diversity. Its trade-off between the complexity and system performance, where the performance of the APT cooperative diversity system is significantly better than the BRS, but less than the MRT. On the other hand, the APT has less complexity at both the relays and the destination since the relay needs to know only its own channels [10], [11]. With out loss of generality, at each relay, the channel state information (CSI) is available, which mean that the relay knows the channels between $(S \rightarrow$ Relays $\rightarrow D)$. Therefore, the weight of the relay $i$ can be expressed as

$$
w_{i}=\frac{1}{\sqrt{M}} \frac{h_{i}^{*}}{\left|h_{i}\right|} \frac{g_{i}^{*}}{\left|g_{i}\right|} .
$$

Therefore, the received signal at the destination can be written as

$$
\begin{aligned}
y_{D}= & \frac{1}{\sqrt{M}} \\
& \sum_{i=1}^{M}\left(\left|h_{i}\right|\left|g_{i}\right| r_{i}^{-\frac{\alpha}{2}} l_{i}^{-\frac{\alpha}{2}} x+\left|g_{i}\right| \frac{h_{i}^{*}}{\left|h_{i}\right|} r_{i}^{-\frac{\alpha}{2}} n_{i}\right)+n_{d} .
\end{aligned}
$$

Thus, the SNR at the destination node is given by

$$
\mathrm{SNR}=\frac{\left(\sum_{i=1}^{M}\left|h_{i}\right| l_{i}^{-\frac{\alpha}{2}}\left|g_{i}\right| r_{i}^{-\frac{\alpha}{2}}\right)^{2}}{\sigma_{r}^{2} \sum_{i=1}^{M}\left|g_{i}\right|^{2} r_{i}^{-\alpha}+M \sigma_{d}^{2}} .
$$




\section{B. Orthogonal Scheme}

To compare the performance of cooperative diversity of multi relays using APT, we also consider an OS that employs orthogonal channel access [12]. Each link $\left(S \rightarrow R_{i} \rightarrow D\right)$, where $i=1, \ldots \ldots ., M$ uses $\frac{1}{2 M}$ channel resources for communication. Without loss of generality, the relay $i$ will receive $y_{i}$, and amplify it with gain $\omega_{i}$. According to CSI assisted amplify and forward (AF) relaying, to satisfy the peak power concentration, the gain of the relay $i$ is given as

$$
\omega_{i}=\sqrt{\frac{1}{\left|h_{i}\right|^{2}+\sigma_{r}^{2}}} .
$$

In the second hop, the relay forwards the signal received from the source. Therefore, the SNR can be written as

$$
\mathrm{SNR}_{i}=\frac{\left|h_{i}\right|^{2} l_{i}^{-\alpha}\left|g_{i}\right|^{2} r_{i}^{-\alpha} \omega_{i}^{2}}{\omega_{i}^{2} \sigma_{r}^{2}\left|g_{i}\right|^{2} r_{i}^{-\alpha}+\sigma_{d}^{2}}
$$

This can be written as

$$
\mathrm{SNR}_{r i}=\frac{\frac{\left|h_{i}\right|^{2} l_{i}^{-\alpha}}{\sigma_{r}^{2}} \frac{\left|g_{i}\right|^{2} r_{i}^{-\alpha}}{\sigma_{d}^{2}}}{\frac{\left|g_{i}\right|^{2} r_{i}^{-\alpha}}{\sigma_{r}^{2}}+\frac{\left|h_{i}\right|^{2} l_{i}^{-\alpha}}{\sigma_{d}^{2}}+1} .
$$

The form of the SNR with all relays participating is

$$
\mathrm{SNR}=\sum_{i=1}^{M} \frac{\frac{\left|h_{i}\right|^{2} l_{i}^{-\alpha}}{\sigma_{r}^{2}} \frac{\left|g_{i}\right|^{2} r_{i}^{-\alpha}}{\sigma_{d}^{2}}}{\frac{\left|g_{i}\right|^{2} r_{i}^{-\alpha}}{\sigma_{r}^{2}}+\frac{\left|h_{i}\right|^{2} l_{i}^{-\alpha}}{\sigma_{r}^{2}}+1} .
$$

\section{Best Relay Selection}

In the above scheme, the performance of the system is degraded because of inefficient use of channel resources. This can be eliminated using the BRS scheme. In this scheme, the relay node which achieves the highest end-to-end SNR at the destination is selected to retransmit the signal from the source to destination. As result, only two channels are required, regardless of the number of relays. Without loss of generality, the end-to-end SNR of $i$ relay can be written as

$$
\mathrm{SNR}_{i}=\frac{\gamma_{1 i} \gamma_{2 i}}{\gamma_{1 i}+\gamma_{2 i}+1}
$$

where $\gamma_{1 i}=\frac{\left|h_{i}\right|^{2} l_{i}^{-\alpha}}{\sigma_{r}^{2}}$ is the instantaneous SNR of the source signal at relay $i$, and $\gamma_{2 i}=\frac{\left|g_{i}\right|^{2} r_{i}^{-\alpha}}{\sigma_{d}^{2}}$ is the instantaneous SNR of the relayed signal (by relay $\stackrel{d}{i}$ ) at the destination. Because the relay used is the one that achieves the highest end-to-end SNR [13], the SNR at the destination node can be written as

$$
\mathrm{SNR}_{\text {Best }}=\max _{i}\left(\frac{\gamma_{1 i} \gamma_{2 i}}{\gamma_{1 i}+\gamma_{2 i}+1}\right) .
$$

\section{Maximal Ratio Transmission}

The other way to achieve cooperative diversity at the relay nodes is MRT, which combats severe fading effects and achieves the maximum SNR [14]. Thus, the weight of the relay $i$ is $w_{i}$ [15], where

$$
w_{i}=\frac{g_{i}^{*}}{\sqrt{\sum_{i=1}^{M}\left|g_{i}\right|^{2}}} .
$$

From (12), the weight of each relay depends on the channel between the relay and destination. Moreover, each relay needs to know all the channels between each relay and destination. This means that the complexity of this scheme will be greater than APT, where each relay needs to know only its own channels. The destination will then constructively combine the received signals from all relays, to obtain the desired information more reliably. Therefore, the received signal at the destination can be written as

$$
\begin{aligned}
y_{D} & =\sum_{i=1}^{M} \\
& \left(\frac{\left|g_{i}\right|^{2}}{\sqrt{\sum_{i=1}^{M}\left|g_{i}\right|^{2}}} h_{i} r_{i}^{-\frac{\alpha}{2}} l_{i}^{-\frac{\alpha}{2}} x+\frac{\left|g_{i}\right|^{2}}{\sqrt{\sum_{i=1}^{M}\left|g_{i}\right|^{2}}} r_{i}^{-\frac{\alpha}{2}} n_{i}\right) \\
& +n_{d} .
\end{aligned}
$$

Therefore, the SNR at the destination node is given by

$$
\mathrm{SNR}=\frac{\left(\sum_{i=1}^{M} \frac{\left|h_{i}\right|\left|g_{i}\right|^{2}}{\sqrt{\sum_{i=1}^{M}\left|g_{i}\right|^{2}}} r_{i}^{-\frac{\alpha}{2}} l_{i}^{-\frac{\alpha}{2}}\right)^{2}}{\sigma_{r}^{2} \sum_{i=1}^{M} \frac{\left|g_{i}\right|^{4}}{\sum_{i=1}^{M}\left|g_{i}\right|^{2}} r_{i}^{-\alpha}+\sigma_{d}^{2}} .
$$

\section{E. Optimum Combining Scheme}

In this section, we obtain the performance of the cooperative diversity based on the optimum combining weights in terms of received SNR at the destination [16], [17]. To achieve the optimum combination of the received signals at the destination, the optimum combining weights $w_{i}$ are given by

$$
w_{i}=\frac{\sigma_{r}^{2}+\left|h_{i}\right|^{2} l_{i}^{-\alpha}}{\sigma_{r}^{2} \sigma_{d}^{2}+\left|g_{i}\right|^{2} r_{i}^{-\alpha} \sigma_{d}^{2}+\left|h_{i}\right|^{2} l_{i}^{-\alpha} \sigma_{r}^{2}} .
$$

Applying the optimum combining weights given in (15), the instantaneous SNR at the destination node is given by

$$
\mathrm{SNR}=\sum_{i=1}^{M} \frac{\frac{\left|h_{i}\right|^{2} l_{i}^{-\alpha}}{\sigma_{r}^{2}} \frac{\left|g_{i}\right|^{2} r_{i}^{-\alpha}}{\sigma_{d}^{2}}}{\frac{\left|g_{i}\right|^{2} r_{i}^{-\alpha}}{\sigma_{r}^{2}}+\frac{\left|h_{i}\right|^{2} l_{i}^{-\alpha}}{\sigma_{d}^{2}}+1} .
$$

\section{Spectral EFFiciency ANALYsis of EGT}

In communication systems, the SE describes the data rate that can be sent over specific bandwidth. In this section, we derive the achievable SE of the two phase relay system, which can be expressed as

$$
\Re=\frac{1}{2} \mathrm{E}\left[\log _{2}(1+\mathrm{SNR})\right]
$$

The factor $\frac{1}{2}$ comes from the fact that the communications between the source and destination is performed in two phases. The achievable SE of the two phase relay system with the EGT scheme can be written as

$$
\Re=\frac{1}{2} \mathrm{E}\left\{\log _{2}\left(1+\frac{\left(\sum_{i=1}^{M}\left|h_{i}\right|\left|g_{i}\right| r_{i}^{-\frac{\alpha}{2}} l_{i}^{-\frac{\alpha}{2}}\right)^{2}}{\sigma_{r}^{2} \sum_{i=1}^{M}\left|g_{i}\right|^{2} r_{i}^{-\alpha}+M \sigma_{d}^{2}}\right)\right\} .
$$


The expectation in (17) is with respect to random variable SNR, where its a ratio of a large number of random variables, and it seems to be difficult to derive a closed-form expression for its distribution. The overall SE of our proposed model can be found from (18), where we need to find the expectation of this equation with respect to the following set of $(4 M)$ random variables: $\mathbf{h}=\left\{h_{1}, h_{2}, \ldots, h_{M}\right\}, \mathbf{r}=\left\{r_{1}^{-\alpha}, r_{2}^{-\alpha}, \ldots, r_{M}^{-\alpha}\right\}$, $\mathrm{g}=\left\{g_{1}, g_{2}, \ldots, g_{M}\right\}$, and $\mathbf{l}=\left\{l_{1}^{-\alpha}, l_{2}^{-\alpha}, \ldots, l_{M}^{-\alpha}\right\}$. The direct approach to compute this expectation is difficult to obtain in general, as it may require the computation of a $(4 M)$ fold convolution integrals. As result, in this paper we use a useful Lemma1 to evaluate the averaging in (18), where can be easily evaluated.

Lemma 1. [18, Eq. (5)] Let $x_{1}, \ldots, x_{N}, y_{1}, \ldots, y_{M}$ be arbitrary random variables $>0$. Then

$$
\begin{aligned}
\ln (1+ & \left.\frac{\sum_{n=1}^{N} x_{n}}{\sum_{m=1}^{M} y_{m}+1}\right)= \\
& \int_{0}^{\infty} \frac{1}{z}\left(1-e^{-z \sum_{n=1}^{N} x_{n}}\right) e^{-z\left(\sum_{m=1}^{M} y_{m}+1\right)} d z
\end{aligned}
$$

Thus, we can rearrange (18) to obtain the exact analytical expression for $\mathrm{SE}$ as follows:

$$
\begin{aligned}
& \Re=\frac{1}{2} \mathrm{E}\left[\left(\log _{2} e\right)\right. \\
& \times \int_{0}^{\infty} \frac{1}{z}\left(1-e^{\left.-z\left(\sum_{i=1}^{M}\left|h_{i}\right|\left|g_{i}\right| r_{i}^{-\frac{\alpha}{2}} l_{i}^{-\frac{\alpha}{2}}\right)^{2}\right)}\right) \\
&\left.\quad \times e^{-z \sigma_{r}^{2} \sum_{i=1}^{M}\left|g_{i}\right|^{2} r_{i}^{-\alpha}} e^{-z M \sigma_{d}^{2}} d z\right] .
\end{aligned}
$$

The expectation in (20) is not easy to obtain, where the square in the exponent includes all the summation. In this regard, we use corollary 2 to simplify the evaluation of the required average, which make the random variables appear only in a liner combination at the exponent.

Corollary 2. [19, Eq. (6)] Recalling firstly the well-known identity

$$
\frac{1}{\sqrt{\pi}} \int_{-\infty}^{\infty} e^{-\omega^{2}} d \omega=1
$$

which is also equivalent to

$$
1=\frac{1}{\sqrt{\pi}} \int_{-\infty}^{\infty} e^{-(\omega+j u)^{2}} d \omega \quad \forall u .
$$

Equation (22) implies that

$$
e^{-z u^{2}}=\frac{1}{\sqrt{\pi}} \int_{-\infty}^{\infty} e^{-\omega^{2}} e^{-j 2 \omega \sqrt{z} u} d \omega
$$

Because the left hand side of (23) is real, it follows that the right hand side should also be real. Thus, (23) can be written as:

$$
e^{-z u^{2}}=\frac{1}{\sqrt{\pi}} \int_{-\infty}^{\infty} e^{-\omega^{2}} \operatorname{Re}\left(e^{-j 2 \omega \sqrt{z} u}\right) d \omega
$$

According to [20], this means (20) can be written as

$$
\begin{aligned}
\Re=\frac{1}{2}\left(\log _{2} \mathrm{e}\right) \int_{0}^{\infty} \frac{e^{-z M \sigma_{d}^{2}}}{z}\left(1-\frac{1}{\sqrt{\pi}} \int_{-\infty}^{\infty} \mathrm{e}^{-\omega^{2}}\right. \\
\times \operatorname{Re}\left(\prod _ { i = 1 } ^ { M } \mathrm { E } \left[e^{\left.\left.\left.-j 2 \omega \sqrt{z}\left|h_{i}\right|\left|g_{i}\right| r_{i}^{-\frac{\alpha}{2}} l_{i}^{-\frac{\alpha}{2}}\right]\right) d \omega\right)}\right.\right. \\
\times \prod_{i=1}^{M} \mathrm{E}\left[e^{-z \sigma_{r}^{2}\left|g_{i}\right|^{2} r_{i}^{-\alpha}}\right] d z .
\end{aligned}
$$

which can be expressed as

$$
\Re=\frac{1}{2}\left(\log _{2} \mathrm{e}\right) \int_{0}^{\infty} \frac{e^{-z M \sigma_{d}^{2}}}{z}\left(1-\mathcal{M}_{v}(z)\right) \mathcal{M}_{u}(z) d z
$$

where $\mathcal{M}_{v}(z)=\frac{1}{\sqrt{\pi}} \int_{-\infty}^{\infty} e^{-\omega^{2}} \operatorname{Re} \Phi(2 \sqrt{z} \omega) d \omega$, and $\Phi(\omega)=$

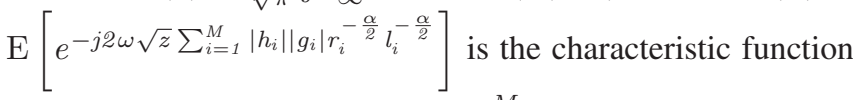
(CHF) of the sum, and $\mathcal{M}_{u}(z)=\prod_{i=1}^{M} \mathrm{E}\left[e^{-z u}\right]$ is the MGF of $u$, where $u=\sigma_{r}^{2}\left|g_{i}\right|^{2} r_{i}^{-\alpha}$. Hence, $\left|g_{i}\right|^{2}$ is the power of the channel gain, which is represented by random variable with exponential distribution. Therefore, $\mathcal{M}_{u}(z)$ can be simplified to

$$
\begin{aligned}
& \mathcal{M}_{u}(z)=\prod_{i=1}^{M} \mathrm{E}\left[e^{-z \sigma_{d}^{2}\left|g_{i}\right|^{2} r_{i}^{-\alpha} \mid r_{i}^{-\alpha}}\right] \\
&=\prod_{i=1}^{M}\left(\frac{1}{1+z \sigma_{d}^{2} r_{i}^{-\alpha}}\right) .
\end{aligned}
$$

The term $r_{i}^{-\alpha}$ stands for the random distance between the relay $r_{i}$ and the destination, as far as the distribution of $r_{i}$ is concerned, it can be shown that their probability density function (PDF) is $f(r)=2 r / A^{2}, r \leq A$. Therefore, (27) can be expressed as

$$
\begin{aligned}
\mathcal{M}_{u}(z)= & \prod_{i=1}^{M} \int_{0}^{A} \frac{1}{1+z \sigma_{d}^{2} r_{i}^{-\alpha}} \frac{2 r_{i}}{A^{2}} d r_{i}= \\
& \left(1-{ }_{2} F_{1}\left(1 ; \frac{2}{\alpha} ; 2+\frac{2+\alpha}{\alpha} ;-\frac{A^{\alpha}}{z \sigma_{d}^{2}}\right)\right)^{M}
\end{aligned}
$$

where ${ }_{2} F_{1}(a ; b ; u)$ is the confluent hypergeometric function. Also, $\mathcal{M}_{v}(z)$ can be simplified as (29), which is shown at the top of next page. Equation (29) gives the MGF of $v$ at arbitrary distances $r$, and $l$, which can be obtained by averaging out $\mathcal{M}_{v}(z)$ in (29) with respect to the distribution of $r$ and $l$, as shown in (28). It is worth mentioning that (29) can also be 
Table I: Simulation Parameters

\begin{tabular}{lc}
\hline Parameter & Value \\
\hline Path loss exponent $(\alpha)$ & 3 \\
Number of relays $(M)$ & 10 \\
Radius area $(A)$ & $1 \mathrm{~km}$ \\
Noise power at relay $\left(\sigma_{r}^{2}\right)$ & 1 watte $/ \mathrm{Hz}$ \\
Noise power at destination $\left(\sigma_{d}^{2}\right)$ & 1 watte $/ \mathrm{Hz}$ \\
\hline
\end{tabular}

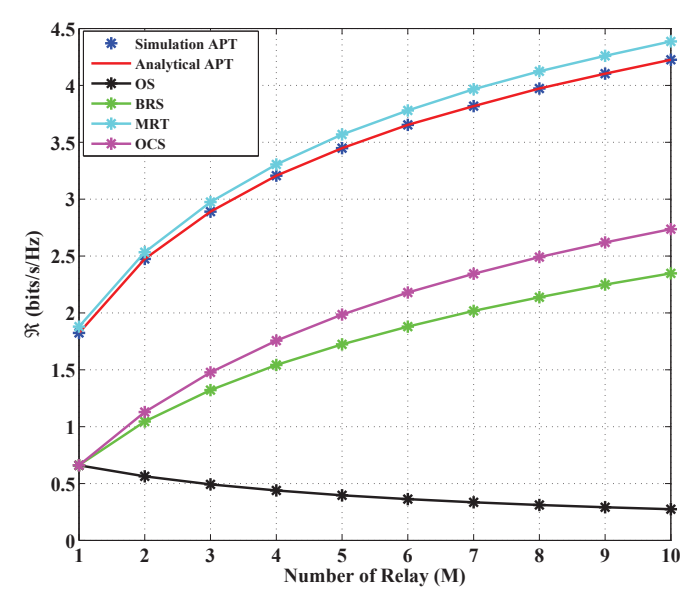

Figure 2: SE as function of $M$.

expressed in terms of the weights and abscissas of a Laguerre orthogonal polynomial, therefore (29) can be expressed as (30), which is shown at the top of next page, where $\lambda_{e}$ and $\Psi_{e}$ are the sample points and the weights factors of the Laguerre polynomial, respectively, tabulated in [21, Eq. (25.4.46)]. The remainder $R_{E}$ is small for $E \geq 15$. Substituting (27) and (30) into (26) gives the final expression of overall SE.

Equation (26) can also be expressed in terms of the weights and abscissas of a Laguerre orthogonal polynomial

$$
\Re=\frac{1}{2}\left(\log _{2} \mathrm{e}\right) \sum_{n=1}^{\mathrm{N}} \frac{\xi_{n}}{\beta_{n}}\left(1-\mathcal{M}_{v}\left(\mu_{n}\right)\right) \mathcal{M}_{u}\left(\mu_{n}\right)+R_{N}
$$

where $\mu_{n}=\frac{\beta_{n}}{M \sigma_{d}^{2}}, \beta_{n}$ and $\xi_{n}$ are the sample points and the weights factors of the Laguerre polynomial, respectively, tabulated in [21, Eq. (25.4.45)]. The remainder $R_{N}$ is sufficiently small for $N \geq 15$; therefore, (31) provides an efficient numerical evaluation method for the required SE.

\section{Numerical AND Simulation Results}

In this section, we provide the numerical and simulation results for the performance of SE as a function of the number of relays, noise power at the relays, and destination. Our analytical results for APT are compared with Monte-Carlo simulation results for verification. We compare cooperative diversity networks which apply different schemes at the relays and the destination nodes.

Fig. 2, shows SE of five different schemes as function of the number of relays, $M$, when $\sigma_{r}^{2}=\sigma_{d}^{2}=1 \mathrm{watt} / \mathrm{Hz}$. We can see that the theoretical APT curve is a good match to the simulated APT performance. We also see that an

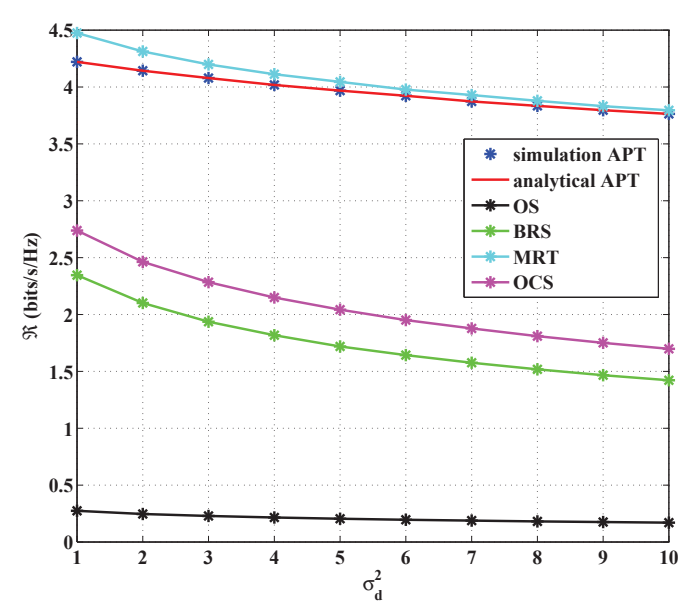

Figure 3: SE as function of $\sigma_{d}^{2}$.

increase in the number of cooperating relay nodes $(\mathrm{M})$ has a beneficial impact on enhancement of the SE performance, and the achieved diversity order for the different schemes, except for OS, where the system performance worsens as M increases. In addition, it is evident that the performance of the APT cooperative diversity system is significantly better than the others, except for the APT. However, the APT needs to know the channels of all the relays, which leads to an increase in the complexity of the system. The APT has less complexity at both the relays and the destination since the relay needs to know only its own channels.

Fig. 3, illustrates the SE as function of the noise power at the destination, when $M=10$, and $\sigma_{r}^{2}=1 \mathrm{watt} / \mathrm{Hz}$. The effect of this noise on the schemes varies. OCS was the most affected by an increase the $\sigma_{d}^{2}$, followed by the BRS, which also decreased with increase in $\sigma_{d}^{2}$. The degradation of the remaining schemes (MRT and APT) was less than for OCS and BRS. In (18), the term $M \sigma_{d}^{2}$ appears in the denominator so as either $M$ or $\sigma_{d}^{2}$ increases, SE will decrease. In addition, any increase in $\sigma_{d}^{2}$ is multiplied by $\mathrm{M}$, which enhances the effect on system performance. The effect of this noise on OS scheme is very small, and it can be negligible.

Fig. 4, compares the performance of the SE for the same five schemes as a function of noise power at the relays, $\sigma_{r}^{2}$, when $\mathrm{M}=10$, and $\sigma_{d}^{2}=1 \mathrm{watt} / \mathrm{Hz}$. It is clear that $\mathrm{SE}$ decreases with increase in $\sigma_{r}^{2}$ for all the schemes. We also notice that, as expected, at high relay noise power, the performance of the systems dropped dramatically. For instance, at $\sigma_{r}^{2}=10 \mathrm{watt} / \mathrm{Hz}$, it can be seen that $\mathrm{SE}$ is dropped approximately by $1,1,1.5$, and $1.25 \mathrm{bits} / \mathrm{s} / \mathrm{Hz}$ for the BRS, OCS, APT, and MRT, respectively. The effect of this noise on OS scheme is very small, and it can be negligible.

\section{Conclusion}

We have analysed the performance of the APT cooperative diversity network using AF relays over independent, and identically distributed Rayleigh fading channels. We also 


$$
\begin{aligned}
& \mathcal{M}_{v}(z)= \\
& \frac{1}{\sqrt{\pi}} \int_{-\infty}^{\infty} e^{-\omega^{2}} \operatorname{Re} \prod_{i=1}^{M}\left(\frac{\left(l_{i} r_{i}\right)^{\alpha} \sqrt{1+4 \omega^{2} z\left(l_{i} r_{i}\right)^{-\alpha}}-j \omega\left(l_{i} r_{i}\right)^{\frac{\alpha}{2}} \sqrt{z}\left(\pi-j 2 \operatorname{Arcsinh}\left(2 \omega\left(l_{i} r_{i}\right)^{\frac{-\alpha}{2}} \sqrt{z}\right)\right)}{\left(\left(l_{i} r_{i}\right)^{\alpha}+4 \omega^{2} z\right) \sqrt{1+4 \omega^{2} z\left(l_{i} r_{i}\right)^{-\alpha}}}\right) d \omega .
\end{aligned}
$$

$$
\begin{aligned}
& \mathcal{M}_{v}(z)= \\
& \frac{1}{\sqrt{\pi}} \sum_{e=1}^{E} \Psi_{e} \operatorname{Re} \prod_{i=1}^{M}\left(\frac{\left(l_{i} r_{i}\right)^{\alpha} \sqrt{1+4 \lambda_{e}^{2} z\left(l_{i} r_{i}\right)^{-\alpha}}-j \lambda_{e}\left(l_{i} r_{i}\right)^{\frac{\alpha}{2}} \sqrt{z}\left(\pi-j 2 \operatorname{Arcsinh}\left(2 \lambda_{e}\left(l_{i} r_{i}\right)^{\frac{-\alpha}{2}} \sqrt{z}\right)\right)}{\left(\left(l_{i} r_{i}\right)^{\alpha}+4 \lambda_{e}^{2} z\right) \sqrt{1+4 \lambda_{e}^{2} z\left(l_{i} r_{i}\right)^{-\alpha}}}\right)+R_{E} .
\end{aligned}
$$

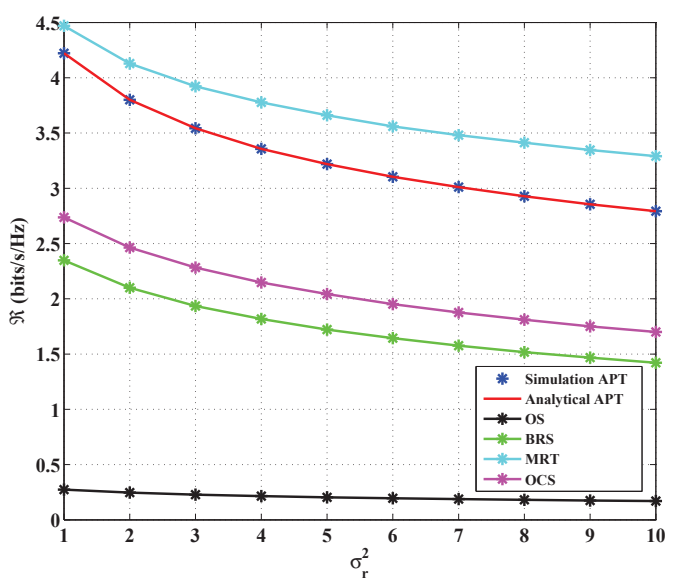

Figure 4: SE as function of $\sigma_{r}^{2}$.

compared the performance of the APT cooperative diversity system with OS, BRS, MRT, and OCS. Using the MGF, an exact-form expression for SE was obtained. The accuracy of the new mathematical results has been confirmed by Monte Carlo simulation. These results show that APT scheme can provide better performance compared with the other schemes, except the MRT. However, the APT benefits from greater simplicity, each relay needs to know only its channels, and the CSI is not required at the destination. Finally, we have shown the effect of variation of important factors such as number of relays, noise power at the relays and noise power at the destination, on predicted system performance.

\section{REFERENCES}

[1] F. Mansourkiaie and M. H. Ahmed, "Per-node traffic load in cooperative wireless sensor networks," IEEE Commun. Lett., vol. 20, no. 2, pp. 344 $347,2016$.

[2] X. Zhang, M. O. Hasna, and A. Ghrayeb, "An adaptive transmission scheme for two-way relaying with asymmetric data rates," IEEE Trans. Veh. Technol, vol. 65, no. 3, pp. 1477-1491, 2016.

[3] P. K. Sharma and P. K. Upadhyay, "Cooperative spectrum sharing in twoway multi-user multi-relay networks," IET Communications, vol. 10, no. 1, pp. 111-121, 2016.
[4] L. Zheng and D. Tse, "Diversity and multiplexing: a fundamental tradeoff in multiple-antenna channels," IEEE Trans. Inf. Theory, vol. 49 no. 5, pp. 1073-1096, 2003.

[5] S. Ikki and M. Ahmed, "Performance of cooperative diversity using equal gain combining (EGC) over Nakagami-m fading channels," IEEE Trans. Commun., vol. 8, no. 2, pp. 557-562, Feb. 2009.

[6] Y. Jing and H. Jafarkhani, "Single and multiple relay selection schemes and their achievable diversity orders," IEEE Trans. Commun., vol. 8 , no. 3, pp. 1414-1423, Mar. 2009

[7] M. Hasna and M.-S. Alouini, "End-to-end performance of transmission systems with relays over Rayleigh-fading channels," IEEE Trans. Wireless Commun., vol. 2, no. 6, pp. 1126-1131, Nov. 2003.

[8] P. Anghel and M. Kaveh, "Exact symbol error probability of a cooperative network in a Rayleigh-fading environment," IEEE Trans. Wireless Commun., vol. 3, no. 5, pp. 1416-1421, 2004

[9] S. Ikki and M. Ahmed, "Performance of cooperative diversity using equal gain combining (EGC) over Nakagami-m fading channels," IEEE Trans. Wireless Commun., vol. 8, no. 2, pp. 557-562, 2009.

[10] H. Fu, M. Crussiere, and M. Helard, "Ber analysis for equal gain transmission in downlink multiuser MIMO systems," IEEE Commun. Lett., vol. 4, no. 5, pp. 533-536, 2015.

[11] M. Maaz, M. Helard, P. Mary, and M. Liu, "Performance analysis of time-reversal based precoding schemes in MISO-OFDM systems," in IEEE Veh. Technol. Conf. (VTC), 2015, pp. 1-6.

[12] S. Chen, W. Wang, and X. Zhang, "Performance analysis of multiuser diversity in cooperative multi-relay networks under Rayleigh-fading channels," IEEE Trans. Commun., vol. 8, no. 7, pp. 3415-3419, Jul. 2009

[13] S. S. Ikki and M. H. Ahmed., "Performance analysis of adaptive decodeand-forward cooperative diversity networks with best-relay selection," IEEE Trans. Commun., vol. 58, no. 1, pp. 68-72, Oct. 2010.

[14] T. Lo, "Maximum ratio transmission," IEEE Trans. Commun., vol. 47, no. 10 , pp. $1458-1461$, Oct. 1999.

[15] H. Wang, S. Ma, and T.-S. Ng, "Cooperative communications system with spatially random relays," in IEEE Int. Symp. Personal, Indoor and Mobile Radio Commun. (PIMRC), Sep. 2009, pp. 1968-1972.

[16] D. Brennan, "Linear diversity combining techniques," Proceedings of the IRE, vol. 47, no. 6, pp. 1075-1102, June 1959.

[17] T. Himsoon, W. Su, and K. Liu, "Differential modulation for multinode amplify-and-forward wireless relay networks," in IEEE Wireless Commun. Netw. Conf. (WCNC), vol. 2, Apr. 2006, pp. 1195-1200.

[18] K. Hamdi, "A useful lemma for capacity analysis of fading interference channels," IEEE Trans. Commun., vol. 58, no. 2, pp. 411-416, Feb. 2010.

[19] - "Accurate performance analysis of OFDM over Nakagami-m fading with nonuniform phase distribution," in IEEE Global Telecommun. Conf. (GLOBECOM), Dec. 2010, pp. 1-5.

[20] _ "Some notes on the fourier transform methods for error-rate analysis of equal-gain combining," IEEE Trans. Commun., vol. 58, no. 12 , pp. 3364-3368, Dec. 2010.

[21] M. Abramowitz and I. A. Stegun, Handbook of Mathematical Functions. New York, NY, USA: Dover, 1965. 